\title{
Hat sich der Balkankrieg auf die psychische Gesundheit der Migranten ausgewirkt?
}

Jokica Vrgoc-Mirkovic

1 Bundesamt für Statistik. Ausländerinnen und Ausländer in der Schweiz. Neuenburg: BfS; 2007.

2 Basic O. Die «Jugos» in der Schweiz. NZZ Fokus Nr. 16. 2003.

Korrespondenz:

Dr. med. Jokica Vrgoc-Mirkovic Clienia Littenheid AG

Privatklinik für Psychiatrie und Psychotherapie CH-9573 Littenheid

vrgoc@gmx.ch

\section{Einleitung: Migranten in der Schweiz}

Die Schweiz gehört mit 1,7 Millionen (18\%) Menschen ausländischer Staatsangehörigkeit zu jenen westeuropäischen Staaten, die nach Luxemburg und Liechtenstein die höchste Zahl an Ausländern beherbergen [1]. Die italienische Bevölkerung ist mit 295000 Personen am stärksten vertreten, gefolgt von den Staatsangehörigen aus Deutschland mit 224300 Personen. Die drittgrösste Gruppe sind die Balkanmigranten, die 2007 erneut rückläufig war. Nahezu ein Fünftel aller Ausländer (22,3\%) ist in der Schweiz geboren, fast die Hälfte dieser Bürger lebt seit mindestens 15 Jahren und 12,2\% sogar seit mindestens 30 Jahren in der Schweiz. Eine Million, also zwei Drittel, haben eine zeitlich unbegrenzte Aufenthaltsbewilligung. Grund für den relativ hohen Ausländeranteil in der Schweiz ist nicht nur eine starke Zuwanderung, sondern auch eine restriktive Einbürgerungspolitik. Die Einbürgerungsquote von 1,4\% im Jahre 1997 ist eine der niedrigsten in Westeuropa. Die Zugehörigkeit zu einem EU-Staat, das Verbot der Doppelbürgerschaft durch den Heimatstaat und die zum Teil hohen Anforderungen halten zahlreiche Ausländer vom Erwerb des Schweizer Bürgerrechts ab.

\section{Migrationsgeschichte des ehemaligen Jugoslawien, Imageverlust einer imaginären Nation}

Schon 1968 schloss die jugoslawische Regierung ein Anwerbeabkommen mit westeuropäischen Ländern ab und jahrzehntelang erfüllte Jugoslawien die Funktion eines Arbeitskräftereservoirs mit kontrollierter Migrations- und Auswanderungspolitik in die industriellen Metropolen Europas. In der Tat funktionierte das System der Rotationsmigration mit den jugoslawischen Arbeitern besser als mit den anderen Nationalitäten. Die tüchtigen Jugoslawen waren hochwillkommen und hielten «damit die Wirtschaft anderer Länder in Gang». In der Folge des Balkankrieges setzten Flüchtlingsbewegungen ein und es wurden ganze Familienverbände in die Emigration getrieben. Zugleich verlor Jugoslawien mit dem Zusammenbruch der Ordnung des kalten Kriegs seine herausgehobene Stellung in Südosteuropa. Die Regionalisierung des Balkans in Klein-

\section{La guerre des Balkans a-t-elle eu une influence sur la santé psychique des migrants?}

Presque simultanément à la guerre des Balkans, I'on a constaté une recrudescence des maladies psychiques chez les ressortissants de l'ex-Yougoslavie vivant en Suisse. Les psychiatres dénommèrent ce phénomène, le «Syndrome des Balkans». En furent touchés les patients qui souffraient d'affections psychosomatiques, en particulier de dorsalgies chroniques. Quelles ont donc été les conséquences de la guerre sur la santé psychique de cette diaspora?

La recherche sur le stress a permis de déterminer un taux de cortisol 35 fois supérieur chez les migrants que dans la population locale. Le fait de devoir se réorienter après avoir émigré augmente la sécrétion d'hormones de stress neurobiologique. Celles-ci entraînent une augmentation du tonus musculaire, ce qui cause entre autres des dorsalgies. La guerre dans le pays d'origine a entrainé une perte d'identité psychique et matérielle chez les migrants et aggravé les processus neurobiologiques déjà présents en cas de stress (nouvelle perte de sécurité, pertes de proches, etc.) de même que toute une série d'autres processus neuro-hormonaux. Dans le cadre d'une prise en charge thérapeutique globale des migrants, la thérapie systémique donne de bons résultats.

staaten vertrieb sie an die Peripherie des europäischen Wirtschaftsraums. Bis in die späten 1980er Jahre bestand der Eindruck, dass die Schweiz mit der Rekrutierung von Jugoslawen «eine glückliche Wahl» getroffen habe [2]. Die Jugoslawen waren hochgeschätzte Mitarbeitende, galten als sehr anpassungsfähig und manche Branchen wie 
das Baugewerbe hätten sich noch mehr von «denen» gewünscht. Heute, mehr als 30 Jahre später, sind die Vorurteile ihnen gegenüber ausgeprägter denn je. Sie stehen auf der untersten Stufe der Beliebtheitsskala in der Schweiz, sind schlecht organisiert und ohne eigene Lobby. «Jugos» gelten heute nämlich als gewalttätig, laut und aggressiv, Eigenschaften, von denen viele Schweizer glauben, dass sie sowohl beim Individuum als auch beim Kollektiv gegeben sind. Was hat zum Imageverlust dieser Migrantengruppe geführt?

\section{Bilanz des Balkankrieges}

Von 1991 bis 1995 ereignete sich ein schrecklicher Bürgerkrieg im ehemaligen Jugoslawien mit Tausenden von Toten, Flüchtlingen und Vertriebenen. Der kroatisch-serbische Sezessionskrieg brachte schätzungsweise 20000 Menschen den Tod. Hinzu kamen in diesem Krieg 90000 Vertriebene in Kroatien und 200000 vertriebene Serben aus Kroatien. Zwischen März 1992 und November 1995 sind im bosnischen Interventionskrieg schätzungsweise 215000 Menschen ums Leben gekommen und 2,7 Mio. Muslime, 800000 Serben und 400000 Kroaten vertrieben worden [3]. Dieser Krieg war der grösste Krieg in Europa seit dem Zweiten Weltkrieg. Der Kriegszustand konnte im Jahre 1995 beendet werden.

Etwa zeitgleich mit dem Balkankrieg stieg die Anzahl psychischer Erkrankungen bei Migranten aus dem früheren Jugoslawien in der Schweiz an. Wie werden die somatoformen und Schmerzstörungen aus psychodynamischer Sicht interpretiert?

\section{Psychodynamische Einordnung somatoformer und Schmerzstörungen}

Aus analytischer Sicht ist die Somatisierung als Folge einer psychischen Abwehrleistung von vor allem unangenehmen Wünschen, Triebimpulsen und Affekten zu verstehen. Sie können nicht akzeptiert werden, und die unbewusste Konvertierung in körperliche Symptome ermöglicht eine emotionale Entlastung.

Die Somatisierung ist als Folge einer Alexithymie zu verstehen. Alexithyme Menschen nehmen oft nicht die eigenen emotionalen Reaktionen auf äussere/innere Konfliktspannungen wahr, sondern bemerken nur selektiv physiologische Begleitreaktionen als eigenständige Ereignisse und schieben sie einer körperlichen Erkrankung zu. Die starke Schonhaltung hat das Ziel, die Verschlechterung zu verhindern, und dadurch verstärkt sich der soziale Rückzug, der eine Chronifizierung der Symptomatik zusätzlich begünstigt [4].
Ein weiterer Aspekt der Somatisierung ist, sie als Folge einer verstärkten Stresswahrnehmung $\mathrm{zu}$ interpretieren. Zahlreiche Menschen neigen dazu, auf innere und äussere Belastungen generell aversiv-emotional zu reagieren und dies in Form körperlicher Symptome auszudrücken. Die körperliche Symptomatik führt oft zu vielen ärztlichen Untersuchungen, die von den Patienten als wichtige Zuwendung und damit auch als narzisstische Aufwertung erfahren werden. $\mathrm{Zu}$ gelassene körperliche Symptome sind die einzig verbleibende - und auch soziokulturell akzeptierte - Möglichkeit, auf die erlittenen Verluste aufmerksam zu machen.

Die Stressforschung hat festgestellt, dass der Kortisolspiegel bei Migranten 36-mal höher ist [5]. Der Zwang zur Neuorientierung im Migrationsprozess geht mit einer erhöhten Ausschüttung von Stresshormonen einher [6]. Wie wirkt sich ein dauerhaft erhöhter Kortisolspiegel auf unserem Körper und auf unsere Muskulatur aus? Sind diese neurobiologischen Prozesse für die idiopatische Schmerzsymptomatik verantwortlich? Wenn ja, wie?

\section{Paradigmawechsel, neue Aspekte der Schmerzsymptomatik aus der Stress- forschung}

Entgegen einer früheren Konzeptualisierung «traumatischer Neurosen», die psychopathologisch und psychodynamisch definiert sind, werden psychische Störungen in den letzten Jahrzehnten zunehmend häufiger durch wichtige neurobiologische Mechanismen identifiziert. Nachfolgend werden einige grundlegende neurobiologische Befunde kurz skizziert [7].

In einer neurobiologischen Perspektive ist zunächst die Unterscheidung zweier Pfade der Verarbeitung eines sensorisch-sensiblen Inputs sowie zweier Arten der Gedächtnisbildung bedeutsam:

- In einer expliziten Informationsverarbeitung garantieren vielschichtige Verbindungen zwischen Thalamus, Neocortex, frontalem Cortex und Hippocampus eine exakte Speicherung von faktischen Wissensinhalten (semantisches Wissen und autobiographische Ereignisse).

- Daneben existiert ein paralleler Pfad, der unmittelbar vom Thalamus zur Amygdala führt. Die Amygdala ist die zentrale Schaltstelle des emotionalen Gedächtnisses. Einfache motorische, viszerale, neuroendokrine und neurochemische Abwehrreaktionen werden über zentrale Amygdalakernkomplexe vermittelt. Diese Prozesse spielen sich primär unbewusst ab, sie gehören dem impliziten Gedächtnis an. trie und Psychotherapie. Berlin. Springer; 1999. S.1303-1385 
Mögliche Veränderungen in der zerebralen Informationsverarbeitung unter Extremstress (Abb. 1):

1. Bei Einwirkung massiver Stressoren wird die thalamische Filterfunktion beeinträchtigt.

2. Die in den neuronalen Schaltkreisen von Thalamus - limbischen Strukturen - Cortex zu lokalisierenden Encodierungsprozesse werden gestört, so dass eine Diskonnektion von emotionalen und autobiographischen, d.h. impliziten und expliziten, Gedächtnissystemen resultieren kann.

3. Die infolge einer Hyperaktivierung der Amygdala zunächst ebenfalls übererregte Hippocampusstruktur desintegriert in ihrer Funktionalität; ihre rückwirkende Hemmung auf die via Amygdala aktivierte Hypothalamus-Hypophysen-Nebennierenrinden-(HPA-)Achse entfällt.

4. Es kommt zu einem abnormen neuronalen Gedächtnis, das bereits durch sensorische Einzelreize, die mit der ursprünglichen Traumasituation in eine (auch zufällige) Verbindung gebracht werden, ausgelöst wird.

Bei diesen Prozessen spielt Noradrenalin eine zentrale Rolle vor allem in der initialen Stressbewältigung. Ausschüttung von Noradrenalin triggert auch den Corticotropin-Releasing-Factor, der seinerseits das Hypothalamus-HypophysenNebennierenrinden-System (HPA-Achse) mobilisiert. Die zentrale noradrenerge Innervierung wird durch periphere sympathische Aktivität unterstützt. Langanhaltende Stressexpositionen können zytotoxische Effekte in der Hippocam-

\section{Abbildung 1}

Schematische Darstellung des Abwehrsystems bei klassischer Konditionierung. Basolaterale Anteile der Amygdala spielen eine zentrale Rolle in der klassischen Konditionierung von aversiven Reizen (blau). Viszerale und motorische Reaktionen werden über zentrale Kerne der Amygdala vermittelt. Informationen aus dem präfrontalen Kortex, dem mediodorsalen Thalamus und ventralen Striatum vermitteln komplexe Hemm- und Kontrolleinflüsse auf diese konditionierten Reaktionen und sind bewusst (grau). [14]

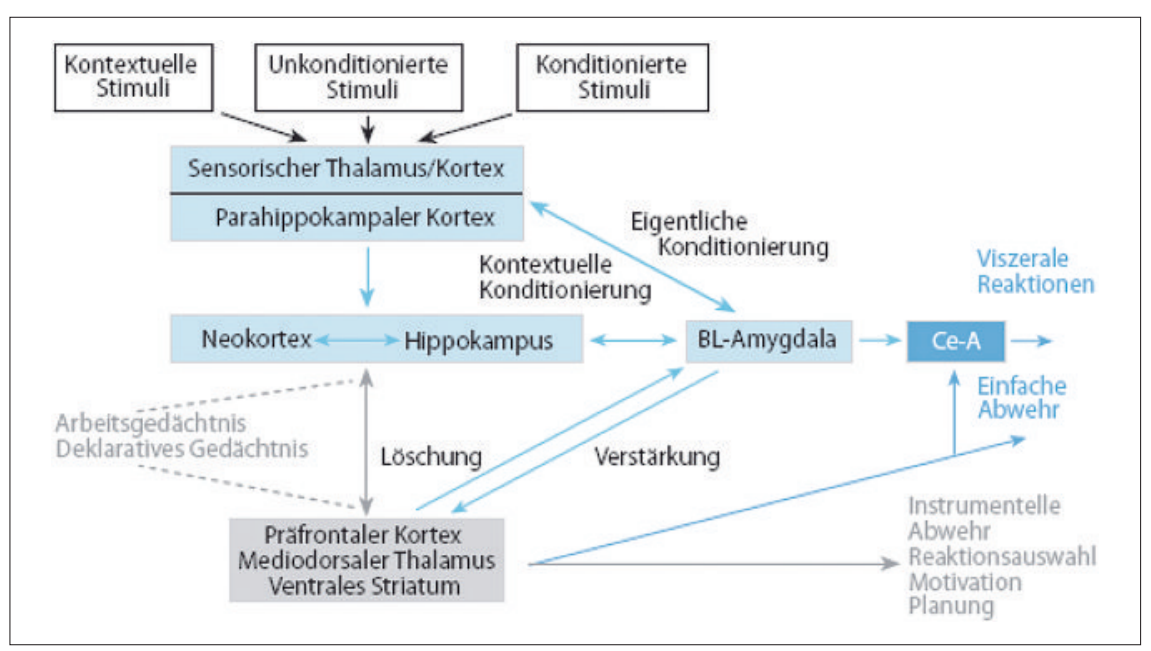

pusformation mit negativen Auswirkungen auf mnestische Funktionen haben. Auf Angst und Flucht reagiert unsere Muskulatur mit einem Beugereflex und einer Tonuserhöhung, die durch kumulativen Stress immer von neuem ausgelöst werden bzw. schliesslich chronisch werden $[7,8]$ und Symptome einer Schmerzstörung oder Fibromyalgie auslösen können.

\section{Fazit}

Da Migranten starkem Migrationstress ausgesetzt sind, der mit hohem Kortisolspiegel einhergeht, entstehen möglicherweise ähnliche neurobiologische Prozesse wie bei PTSD-Patienten. Der Prozess des andauernden Stresses wird im «impliziten Gedächtnis» gespeichert und auf der Ebene des «Körpergedächtnisses» durch Vermeidung und Schonhaltung im Sinne des spontanen traumakompensatorischen Schemas reguliert [9]. Während der afferente motorische Flügel «eingefroren» wird, speichern die efferenten sensomotorischen Flügel die Wahrnehmungs-/Erinnerungsfragmente. Bei einer Abwehrhaltung, die in Sich-Kleinmachen oder Sich-Ducken besteht, kann über Tonuserhöhung in der Nackenmuskulatur der Nervus phrenicus sein direktes Innervationsgebiet auf andere Organe wie das Zwerchfell fehlinnervieren, so dass die Atemexkursionen eingeschränkt und Thorax-, Atem- und Herzbeschwerden ausgelöst werden [9]. Die Schmerzsymptomatik bei Migranten ist nicht nur im Kontext des Migrationsstresses zu verstehen. Hier sind weitere Aspekte aus der eigenen Biographie wie emotionale Vernachlässigung, körperliche und/oder sexuelle Gewalt, Armut, psychische oder Suchtkrankheiten in der Familie, Unfälle usw. mitbeteiligt [4]. Traumatische Erfahrungen in der Kindheit, Migrationsstress und die Kriegsereignisse in der Heimat und damit einhergehende seelische, materielle und Identitätsverluste haben den bereits bestehenden inneren Stress noch verstärkt und damit die obenbeschriebene Reihe der neurohormonellen Prozesse zugespitzt. Diese sind vielleicht als ein letzter Tropfen, der ein volles Fass zum Überlaufen bringt, zu verstehen.

Der Statusverlust, die Enttäuschungen sowie auch das fehlende Identifikationsobjekt (Heimat, Nation) dürfen hier als zusätzliche Faktoren der Dekompensation eines Individuums und seiner Copingfähigkeiten verstanden werden. Migration macht nicht krank, jedoch je grösser der politische, wirtschaftliche und gesellschaftliche Druck zu einseitiger Integration (Assimilation) im Ankunftsland ist, desto höher ist der Stress und die Wahrscheinlichkeit psychischer Beschwerden bei Migranten. Geringfügige individuelle Ressourcen (niedriges Bildungsniveau, schwache Kom- 
10 Minuchin S. 1976

11 Minuchin S. Familie und Familientherapie. Freiburg i.B.: Lambertus; 1971.

12 Oesterreich C. Systemische Therapie an den Grenzen unterschiedlicher kultureller Wirklichkeiten. In: Heise T (Hrsg.). Transkulturelle Psychotherapie. Berlin: VWB; 1998. S 143-58.

13 Pavkovic G. Interkulturelle Beratungskonstellation in der psychosozialen Arbeit. In: Nestmann F, Niepl T (Hrsg.). Beratung von Migranten. Berlin: VWB; 1993; S. $145-63$.

14 Kapfhammer H-P. Anpassungsstörungen, akute und posttraumatische Belastungsstörungen. In: Möller H-J, Laux G, Kapfhammer H-P (Hrsg.). Psychiatrie und Psychotherapie. Band 2. 3. Auflage. Berlin: Springer; 2008. S. $659-722$

- Hoffmann SO. Somatisierungsstörungen und somatoforme Störungen. Theoretisches Verständnis und therapeutische Praxis. Stuttgart: Schattauer; 1998 munikationsfähigkeit usw.), belastende Lebenssituation, leistungsorientierter Lebensstil sowie zuvor erlebte traumatische Erfahrungen in der Kindheit sind begünstigende Faktoren für die psychische Erkrankung.

\section{Systemische Aspekte der transkulturellen Psychiatrie und Psychotherapie}

Die systemische Vorgehensweise [10] basiert auf Systemtheorien, die vor allem aus dem kybernetischen Verständnis der Familienbeziehungen entstanden sind. Der Mensch ist kein isoliertes Wesen, sondern ein agierendes und reagierendes Mitglied einer sozialen Gruppe. Die Systemtherapie zielt auf die Modifizierung der Gegenwart, und systemisches Denken bedeutet auch das Sprechen in Gegensätzen, der sogenannte systemische «Antagonismus». Dabei schliessen sich die beiden Pole nicht aus, sondern bilden eine Ergänzung, verbinden sich zu einem Ganzen: Synthese statt Analyse.

\section{Spezifische Merkmale der Systemtherapie in der Behandlung von Migranten}

Therapeutische Arbeit mit der Familie ist besonders bei Migranten von Bedeutung. Wie lässt sich diese Aussage bekräftigen?

Migration führt zum Verlust wichtiger Bezugspersonen und zwingt die Familienmitglieder, sich gegenseitig noch stärker zu unterstützen, um die Veränderungen in der Aussenwelt erträglich zu machen. Es kommt hinzu, dass sich der Familienkreis durch die Auswanderung bedeutend verkleinert. Dies führt dazu, dass die Mehrzahl der Migranten noch dichter in Familienbezüge eingebunden ist. Besonders traditionelle Gesellschaften sehen die Gemeinschaft - insbesondere die Familie - als zentrale Einheit, als gemeinsames Ganzes an [11]. So erscheint besonders wichtig, die kulturellen Rahmenbedingungen und die folgenden Grundprinzipien zu beachten [12]:
1. Die psychische Erkrankung eines Familienmitgliedes ist keine Privatangelegenheit, sondern betrifft alle Familienmitglieder.

2. Veränderungen sind generell nur möglich, wenn sie von der Gruppe akzeptiert werden.

3. Bei manchen Kulturen ist entscheidend, ob sich die Schlüsselperson, die den grössten Einfluss auf den Kollektivverband ausübt, auf die Behandlungsvorstellungen einlassen kann.

Diese Ansätze bedeuten nicht, dass man als Therapeut die Vorstellungen und Orientierungen der eigenen Kultur aufgeben muss, sondern es wird die Notwendigkeit von Offenheit hervorgehoben, um den Normen und Werten fremder Kulturen gerecht werden zu können [5, 13]. Die Fähigkeit zur Relativierung der eigenen Werte ist eine Voraussetzung für die interkulturelle Arbeit. Dadurch wird ermöglicht, Brücken zu bauen, über die auch andere gehen können. Besonders bewährt sich die Anwesenheit der erwachsenen Kinder, weil sie meistens durch ihre Integration in der Gesellschaft ein gutes Verständnis für die hiesigen Zusammenhänge haben. In der traditionellen Gesellschaft (z. B. der albanischen, türkischen) steht die Funktion der Familie im Vordergrund. Die sogenannte Ich-Stärke hingegen (z. B. Ablösung) wird als Identitätsverlust erlebt und ist damit eher unerwünscht.

\section{Physiotherapeutischer Ansatz bei Schmerzpatienten}

Für die Behandlung von PTSD- und Schmerzpatienten eignet sich besonders die Myoreflextherapie. Wie oben beschrieben, ist bereits im Ruhezustand in der Muskulatur eine hohe Spannung vorhanden, die der Therapeut provoziert und dabei unter anderem durch Kompression eine Überspannung herbeiführt. Es wird ein Regelkreis in Gang gesetzt, und diese Überspannung wird durch Impulse ans Rückenmark (Reflexfunktion) und von dort ans Gehirn weitergeleitet [8]. 